\title{
3D Printing and Characterization of the Biodegradable Vascular Stent
}

\author{
Zewei Xu, Zongan Li*, Jianping Shi, Liya Zhu, Chunmei Feng, Zhe Yuan \\ Jiangsu Key Laboratory of 3D Printing Equipment \& Manufacturing, Nanjing Normal University, China. \\ *Corresponding author. Tel.: +86-13675107447; email: Zongan_li@njnu.edu.cn \\ Manuscript submitted June 10, 2018; accepted September 14, 2018. \\ doi: 10.17706/ijcee.2018.10.4.254-264
}

\begin{abstract}
Vascular stent is an important kind of medical device for the treatment of the vessel occlusion. In this work, we designed and fabricated a kind of biodegradable vascular stent. The dynamics of expanding, compressing and bending process for the single "sine", "60 degrees sine" and "U" type stent unites were analyzed with Finite Element Method (FEA) respectively, and furthermore, the expanding characters of polylactic acid (PLA) stent models with length of $4 \mathrm{~mm}, 6 \mathrm{~mm}$, and $8 \mathrm{~mm}$ were simulated. The "sine" type bracket had a better dynamic property and thus was more suitable for medical stent implantation in terms of mechanical properties. Then, the stent errors including the movement, the nozzle deposition and the shrinkage of the material were analyzed which provided experimental basis for the designing of the stent. The brachial artery stent implantation on pig experimental animals was carried out using the 3D printed PLA vascular stents. Results showed that the 3D printed vascular could be transported into the stenosis place and expand the vessel after pulling out of the balloon. The blood supply function was normal and the biodegradation could be observed after 30 days. This paper provided a novel method for clinical transformation of biodegradable vascular stents.
\end{abstract}

Key words: Vascular stent, 3D printing, PLA, FEA.

\section{Introduction}

Vascular interventional therapy is gradually becoming the first choice for the treatment of peripheral arterial disease (PAD) with the advantages of minimally invasion, rapid improvement of symptoms and rapid recovery [1]. Therefore, the study of vascular stent forming technology for PAD is becoming more and more important. Biodegradable polymer scaffolds are made from biodegradable or absorbable materials with good histocompatibility and biodegradability. After being implanted, it can effectively dilate the blood vessels in the stenosis, and the thrombosis, foreign body reaction and neointimal hyperplasia are reduced, and it can be degraded gradually, and this material is only hydrolyzed in organisms and eventually produces $\mathrm{CO}_{2}$ and $\mathrm{H}_{2} \mathrm{O}$, and is discharged through blood circulation without toxic effects. Biodegradable polymers have good prospects in the preparation of vascular scaffolds.

Three dimensional printing biodegradable vascular stent technology has obvious advantages over traditional processing technology [2]. 3-dimensional printing technology (3D printing) is an additive manufacturing technology that can control the distribution of materials, reduce product development costs, and shorten product development cycles. Therefore, relying on 3D printing technology, it is of great significance to carry out digital design and manufacturing research on biodegradable polymer stents. 
The first case of the world's first walnut pinch syndrome extravascular stent implantation was completed in the Tangdu Hospital, and the porous titanium alloy extravascular stent was prepared by 3D printing technology. Liu Qing [3] applied for a method and a device for preparing a 3D porous tubular stent, and prepared a 3D porous tubular stent by a 3D printing method, but the stent structure is relatively single. Park [4] used a 3D printing technique to prepare tubular PCL scaffolds to study the effects of drug coating and non-drug coated PCL stent implantation and stent degradation in vitro. The surface morphology and mechanical properties of the stent mainly depend on the molding process of the stent. The personalized design and manufacturing methods make the 3D printing technology has a good application prospect in the preparation of the stent, but in order to obtain a good bending performance, the stent with higher radial support strength needs to be further researched for the 3D molding process.

In this paper, a novel 3D printing method for bio degradable blood vessel stents was proposed. Firstly, we modified an open source fused deposition molding (FDM) 3D printer. And then the CAD vascular stent was designed and analyzed with FEA method to obtain the biomimetic mechanical performance. The influence of the vessel stent fabrication experimental parameters on the mechanical properties was experimental studied. And finally, the vessel stent was implanted into the animal stenosis of vascular lesions.

\section{Method}

\subsection{Vascular Stent Forming Device}

In this paper, a novel 3D printing process for degradable blood vessel stents based on fused deposition molding is used. The basic principle is shown in Fig. 1. The principle of forming is to convert the simple plane motion into a composite motion combining rotation and plane, and the planar structure is processed into a corresponding asymmetrical 3D rotating structure to achieve the requirements of the vascular support. The molding system is divided into several parts such as a melt extrusion nozzle, a molding rotary shaft, and a 3D movement structure. The specific implementation manner is that a hot melt nozzle compresses and deposits semi-fluid materials on a rotating shaft according to a CAD layered data control path. The designated location solidified to form the entire prototype or part.

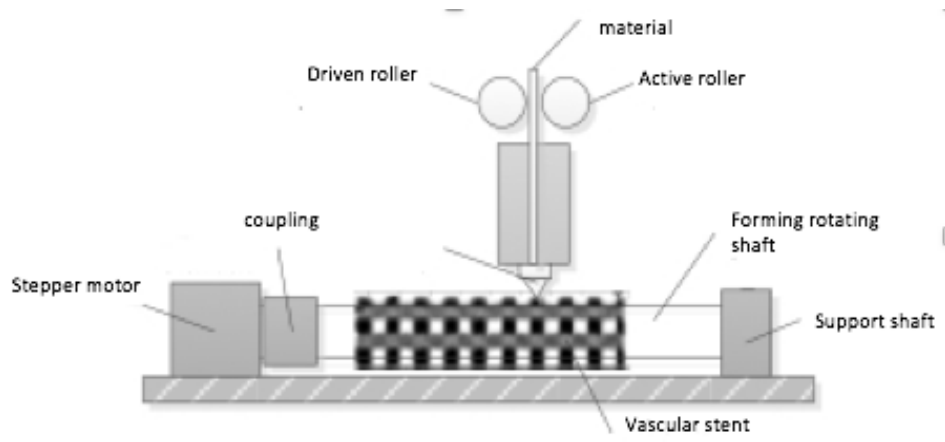

Fig. 1. Schematic diagram of degradable stent formation.

\subsection{Finite Element Analysis of Expansion Process of Vascular Stent Annular Support}

At present, most of the clinically used tubular stents are structural-vascular stents. This tubular structure is formed by bonding adjacent annular supports through connectors. Clinical studies have shown that the balloon dilatation pressure is the main cause of stent restenosis [5]. Therefore, the stent expansion property not only affects the choice of balloon, but also relates to the success rate of operation. By analyzing the working principle of the tubular structure stent, the effect of the expansion performance of the annular support with different structures and the influence of the expansion performance of the annular support 
with different widths are discussed by FEA method, and the whole performance of the tubular support is further optimized.

\subsection{Vascular Stent 3D Printing Process}

1) Through the 3D modeling software SolidWorks 2012 to establish the two-dimensional plane of the stent to expand the 3D graphics, save the file as STL format [6];

2) The saved STL file is imported into the slicing software, the path is generated, and then the generated path is exported from the slicing software and stored as the identifiable G-code of the blood vessel stent forming device.

3) According to the influence of different 3D printing process parameters on the fabricating quality of the vascular stent, the optimum parameters of the $3 \mathrm{D}$ printing molding were determined, and the biodegradable polymer material PLA was used for printing.

\section{Result and discussion}

\subsection{Influence of Expansion Performances}

In order to reduce the amount of computation and improve the simulation efficiency and simplify the support model, the single ring support is taken as the research object without considering the existence of support connections. The finite element computational mesh models for the single annular supports with three structures as shown in Fig. 2(a) "sin" type, (b) "radius $60^{\circ}$ sine" type, and (c) "U" type were respectively established to define the model. The diameter was $5 \mathrm{~mm}$, rib width $0.5 \mathrm{~mm}$, rib thickness $0.2 \mathrm{~mm}$, and bracket width $4 \mathrm{~mm}$.

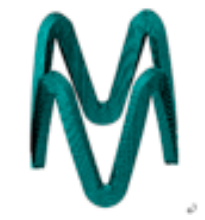

(a)

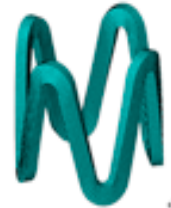

(b)

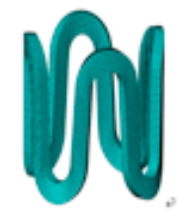

(c)

Fig. 2. Finite element network calculation model.

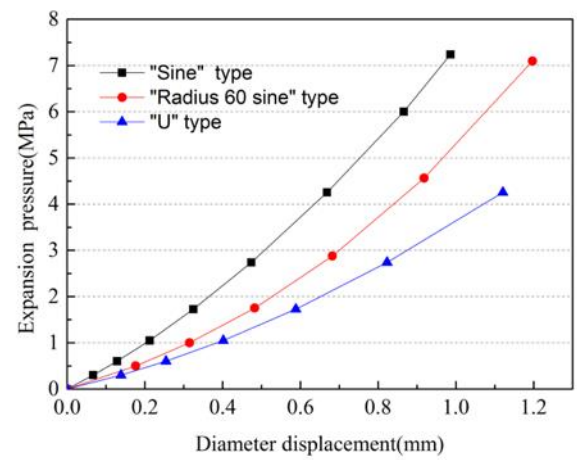

Fig. 3. Relationship between expansion pressure and diameter displacement in annular support with different structures.

The expansion pressure is applied inside the support ring support to expand from original $5 \mathrm{~mm}$ to $6 \mathrm{~mm}$ and then unload. Fig. 3 shows the relationship between the pressure and the displacement of the expansion diameter on the annular support of different structures. Results showed that during the expansion of the 
annular support, the "sine" annular support required a larger expansion pressure when expanding to the same diameter displacement. When the original diameter of the annular support expands to $6 \mathrm{~mm}$, the "sine" type ring support needs an internal pressure of 7.36MPa, the "radius 60 sine" type ring needs 5.23MPa, and the "U" type needs 3.52MPa. The data showed that the ring support structure or the curvature of the ring support arc has a significant impact on stent expansion. The FEA mechanical results using three kinds of ring-shaped structures were showed in the Fig. 4, and when the support is deformed, the deformation position is mainly concentrated at the turn of the annular support, the smaller the bend at the corner, the greater the bending moment bears.
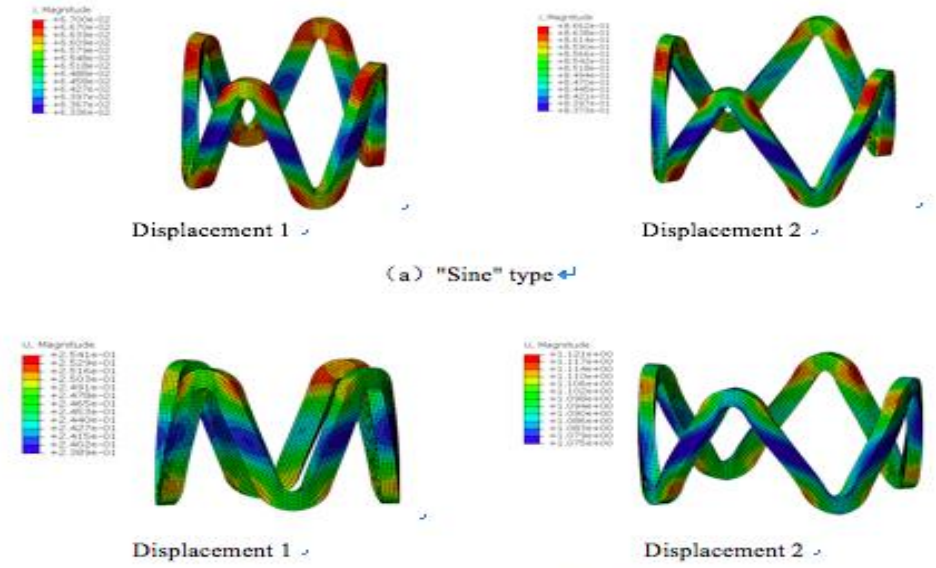

(b) "Radius $60^{\circ}$ Sine" types
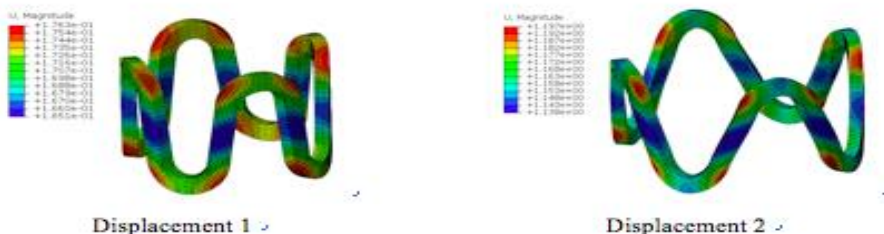

(c) "U" type ,

Fig. 4. Expansive simulation cloud diagram of ring support with different structures.

\subsection{Influence of Expansion Performance of Ring Supports with Different Widths}

Taking the "sine" type single ring support body as the research object, the finite element calculation grid model of single ring support with different width is set up. The width of SH is defined as $4 \mathrm{~mm}, 6 \mathrm{~mm}, 8 \mathrm{~mm}$, and the other parameters and settings are the same, as shown in Fig. 5.

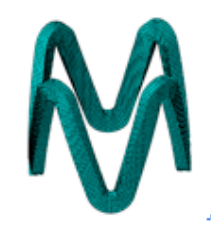

$\mathrm{Sh}=4 \mathrm{~mm}$ 。

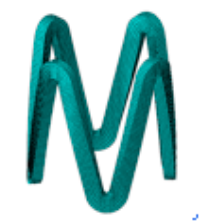

$\mathrm{Sh}=6 \mathrm{~mm}$.

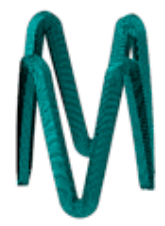

$\mathrm{Sh}=8 \mathrm{~mm}$ 。

Fig. 5. Finite element computational grid model for annular supports with different widths.

The relationship between the pressure and expansion displacement on the radial direction of the annular support with different width was showed in the Fig. 6 . With the same type of structures, when the bracket 
expands the same displacement, the bracket with larger width requires smaller internal pressure. The width of the ring support effects the expansion pressure of the support.

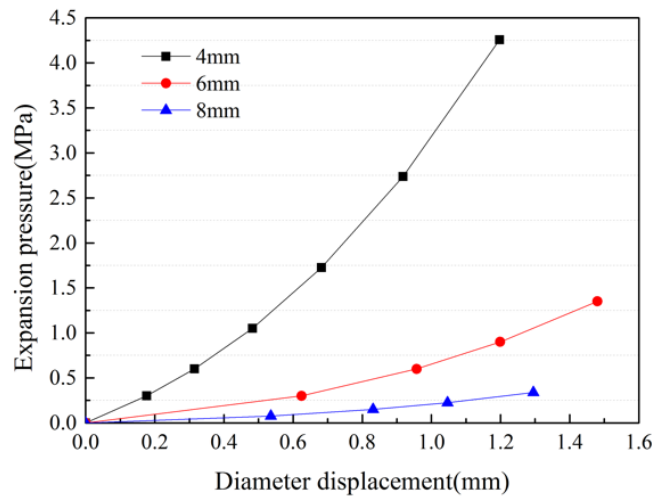

Fig. 6. Relationship between expansion pressure and diameter displacement in annular support with different widths.

It can be seen from Fig. 7, when the ring supports of different widths are subjected to expansion force, the smaller the width of the ring support is, the shorter the area that provides the local deformation is. Based on the theory that the torque is equal to the product of the force and its arm. The shorter the ring-shaped support body, the greater the force required for plastic deformation. In addition, for the smaller width of the annular support, the local plastic deformation zone at the corner is transformed from bending movement to pulling movement, which leads to that the greater force is needed for the local deformation. Therefore, in the stent design process, the selection of an annular support with too narrow width should be avoided.

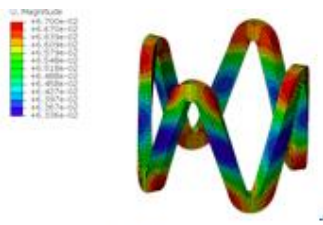

Displacement 1

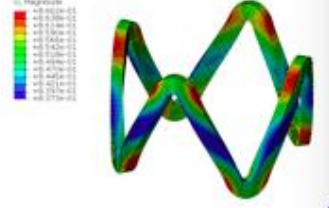

Displacement 2,

(a) $s h=4 \mathrm{~mm}$,
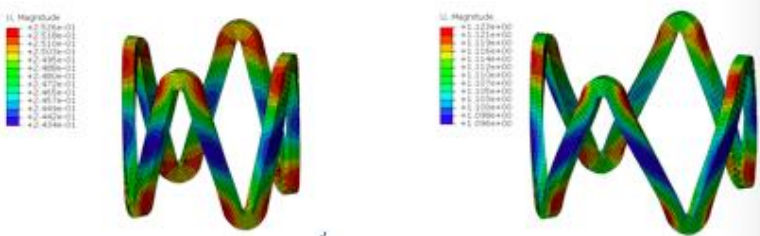

Displacement 1

Displacement 2 ,

(b) $s h=6 \mathrm{~mm}$,

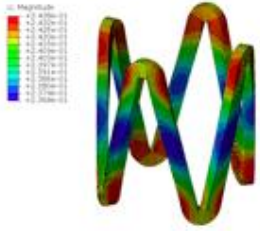

Displacement 1 ,

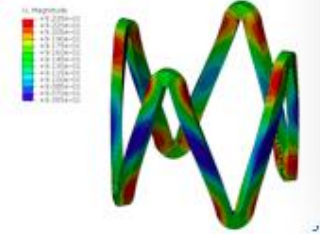

Displacement 2,

(c) $s h=8 \mathrm{~mm}$,

Fig. 7 Simulated cloud expansion of annular support with different widths. 


\subsection{The Effect of System Parameters on the Quality of Stent Formation}

There are many factors that affect the quality of stent formation, which includ nozzle geometry, nozzle temperature, shaft speed and nozzle volume. The forming quality of vascular stents is mainly manifested in microscopic morphology and mechanical properties. The mechanical properties mainly include the radial support and flexibility of the support. Different parameter settings affect the mechanical properties of 3D scaffolds of biodegradable vascular scaffolds. The effects of system parameters on the quality of stent were studied, and this provided experimental basis for 3D printing of biodegradable stent.

\subsubsection{The effect of nozzle aperture on the quality of vascular stent}

All the experiments were at the room temperature. The biodegradable scaffold molding device was used as the experimental platform. The filamentous PLA was selected as the scaffold molding material, and the outlet diameter of the printing nozzle $d$ was $0.4 \mathrm{~mm}$ and $0.2 \mathrm{~mm}$ [7], [8]. The printing nozzle temperature $\mathrm{T}$ was 200 degrees, and the printing speed $\mathrm{F}$ was $400 \mathrm{~mm} / \mathrm{min}$. The preparation of the stent size and setting parameters was shown in Table 1.

Table 1. Vascular Stent Size Parameters

\begin{tabular}{|c|c|c|c|c|c|c|}
\hline structure & $\mathrm{d}(\mathrm{mm})$ & $(\mathrm{b}) \mathrm{mm}$ & $\mathrm{h}(\mathrm{mm})$ & $\begin{array}{c}\text { Connector } \\
\text { width } \\
(\mathrm{mm})\end{array}$ & $\begin{array}{c}\text { Ring } \\
\text { support } \\
\text { width }(\mathrm{mm})\end{array}$ & $\begin{array}{c}\text { Number of } \\
\text { ring } \\
\text { supports }\end{array}$ \\
\hline "Sine" & 5 & 0.5 & 0.2 & 5 & 4 & 10 \\
\hline
\end{tabular}

Five stents were printed with the modified 3D printer of $0.4 \mathrm{~mm}$ and $0.2 \mathrm{~mm}$ nozzles. The radial support force and flexibility of the five stents were tested. The result data of each stent was recorded. The average value of each set of data was calculated. The curve of the relationship between the radial displacement and the support force was plotted as shown in the Fig. 8(a). The forming property of the support is affected by the geometric size of the nozzle. The radial support force of the nozzle with diameter of $0.4 \mathrm{~mm}$ is larger than that of the nozzle with diameter of $0.2 \mathrm{~mm}$. But the compliance of $0.4 \mathrm{~mm}$ is lower than that of $0.2 \mathrm{~mm}$.

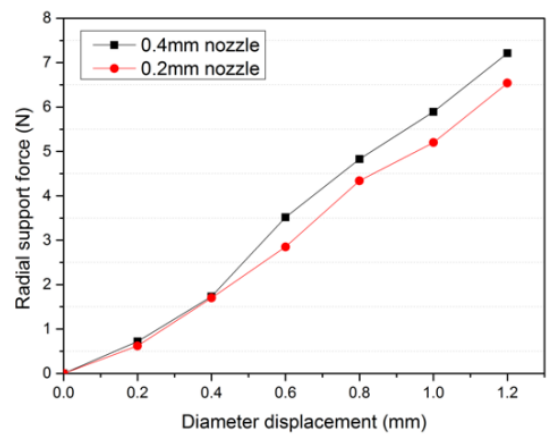

(a) The relationship between the radial support force and the diameter displacement of the support

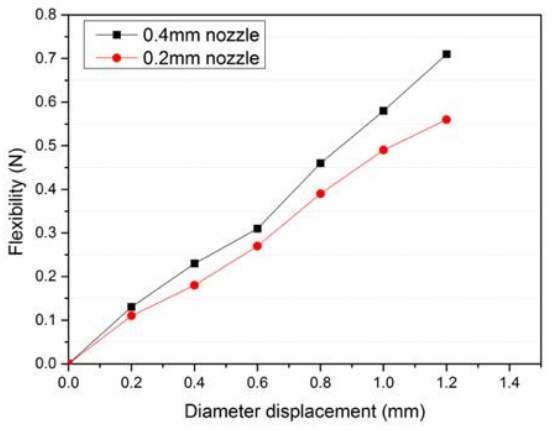

(b) Support flexibility and displacement relation curve

Fig. 8. Effect of nozzle aperture on quality of stent. 
In Table 2, the size of the bracket printed with the $0.4 \mathrm{~mm}$ and $0.2 \mathrm{~mm}$ nozzle is measured. The actual measurements of the bracket are generally larger than the designing result, and the bending moment at the corner of the braced support is the inertia moment of the section. According to the formula $I=b^{3} h / 12$, the width(b) and thickness(h) of the support affect the mechanical properties of the scaffold directly. According to the data in the Table 2, the width and thickness of the supporting ribs made by the nozzle with diameter of $0.4 \mathrm{~mm}$ are generally larger than that of the nozzle with diameter of $0.2 \mathrm{~mm}$.

Table 2. Actual Measurements of Stent Dimensions

\begin{tabular}{|c|c|c|c|c|c|l|}
\hline \multirow{2}{*}{$\begin{array}{c}\text { Serial } \\
\text { number }\end{array}$} & \multicolumn{5}{|c|}{ Geometry of nozzle (mm) } \\
\cline { 2 - 7 } & \multicolumn{3}{|c|}{0.4} & \multicolumn{3}{c|}{0.2} \\
\cline { 2 - 7 } & $\mathrm{b}(\mathrm{mm})$ & $\mathrm{h}(\mathrm{mm})$ & $\mathrm{L}(\mathrm{mm})$ & $\mathrm{b}(\mathrm{mm})$ & $\mathrm{h}(\mathrm{mm})$ & $\mathrm{L}(\mathrm{mm})$ \\
\hline 1 & 0.53 & 0.25 & 46.22 & 0.52 & 0.25 & 46.36 \\
\hline 2 & 0.56 & 0.28 & 46.34 & 0.61 & 0.37 & 46.58 \\
\hline 3 & 0.52 & 0.23 & 46.29 & 0.54 & 0.28 & 46.39 \\
\hline 4 & 0.64 & 0.41 & 46.52 & 0.57 & 0.34 & 46.47 \\
\hline 5 & 0.58 & 0.38 & 46.41 & 0.55 & 0.31 & 46.43 \\
\hline
\end{tabular}

\subsubsection{Effect of nozzle temperature on the quality of vascular stent}

In the actual 3D printing experiment, when the nozzle temperature is too low, the material can not be bonded to the forming rotating shaft, or even cause blockage of the nozzle. When the nozzle temperature is too high, the material will turn to liquid when extruded, so the filamentous extrusion is crucial for the 3D printing [9], [10]. The influence of the temperature of the nozzle on the quality of the scaffold was studied. According to the temperature performance of the PLA, the temperature of the nozzle was set at $\mathrm{T}=200,210$, 220 and 230.

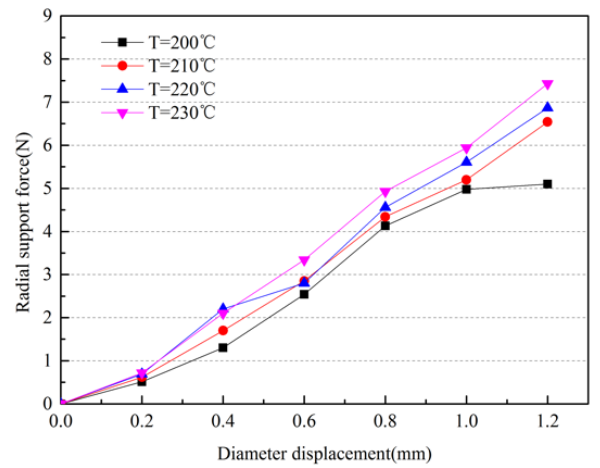

(a) The relationship between the radial support force and the diameter displacement of the support

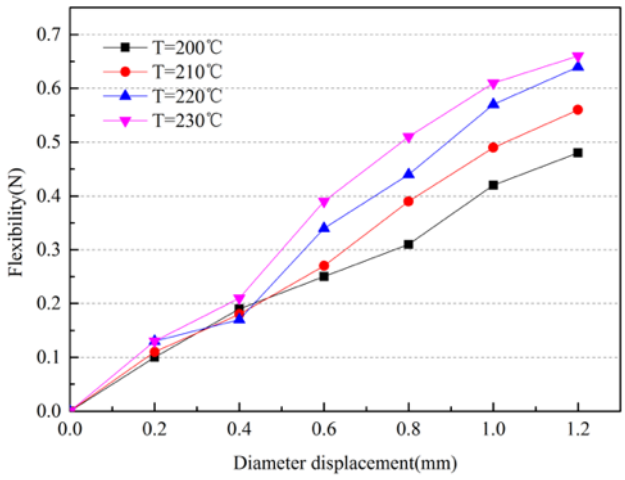

(b) Support flexibility and displacement relation curve

Fig. 9. Effect of nozzle temperature on molding quality of stent. 
Experimental conditions: at the same room temperature, the print nozzle outlet diameter $\mathrm{d}$ was $0.2 \mathrm{~mm}$. The operating speed of the shaft $\mathrm{F}$ was $400 \mathrm{~mm} / \mathrm{min}$. The parameters of the stent was shown in Table 1 . Five stents were printed under each temperature group, and the measurement data of each stent was recorded. Finally, the average value of each group of data was calculated. The displacement curve resulting from the vertical force and its corresponding direction displacement is shown in Fig. 9.

When the nozzle temperature is $\mathrm{T}=210$ degrees, the radial support force and flexibility of the scaffold are closer to the simulated value. When the nozzle temperature $\mathrm{T}=200^{\circ} \mathrm{C}$ and $230^{\circ} \mathrm{C}$, although the prepared stent satisfies the requirements in terms of mechanical properties, the surface of the stent is rough. Especially at $\mathrm{T}=230^{\circ} \mathrm{C}$, a large number of bubbles are distributed on the surface of the stent.

\subsubsection{The effect of printing speed on the quality of vascular stent}

Experimental conditions: at the same room temperature, the print nozzle outlet diameter $\mathrm{d}$ was $0.2 \mathrm{~mm}$. The nozzle temperature is $T=210$, the printing speed is $F=300 \mathrm{~mm} / \mathrm{min}, 400 \mathrm{~mm} / \mathrm{min}$, and $500 \mathrm{~mm} / \mathrm{min}$, and the parameters of the stent are with the same of Table 1, [11], [12]. Five stents were printed under each speed gradient, and the measurement data of each stent was recorded. The displacement curve resulting from drawing the vertical force of the stent in its corresponding direction is shown in the Fig. 10.

Under the mentioned experimental conditions, when the bracket compresses the same displacement, the greater the printing speed $\mathrm{F}$ is, the smaller the radial supporting force is and the better the compliant performance is. It is known from the numerical comparison that when the printing speed is $\mathrm{F}=400 \mathrm{~mm} / \mathrm{min}$, a fully structured tubular support can be prepared, and its radial support performance and flexibility can be kept in a certain equilibrium range. Reasonable setting of printing speed can control the shape and size of extruded wire, thereby improving the micro morphology and mechanical properties of the scaffold.

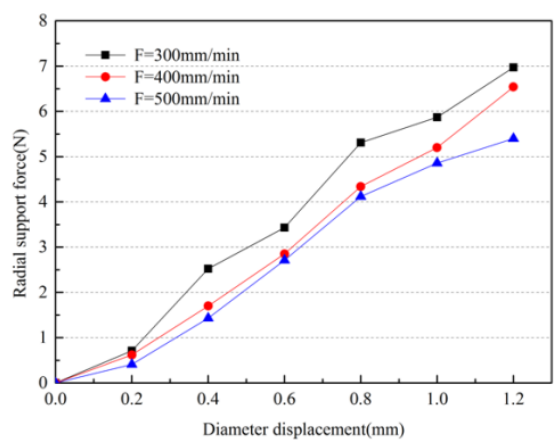

(a) The relationship between the radial support force and the diameter displacement of the support

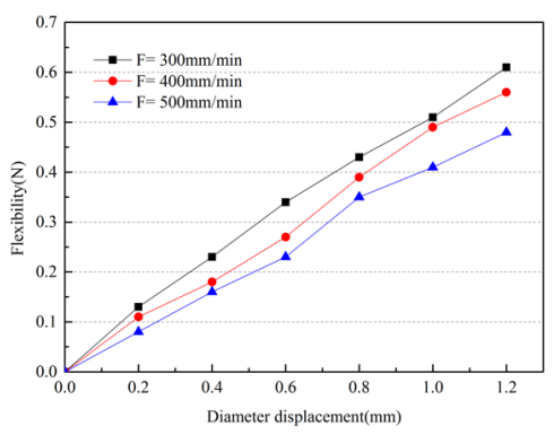

(b) Support flexibility and displacement relation curve

Fig. 10. Effect of print speed on the quality of stent formation.

According to the influence of different 3D printing process parameters on the molding quality of vascular stent, the optimum parameters of 3D printing and forming of vascular stent are obtained. The specific 
parameters are as follows: the diameter of the nozzle $d=0.2 \mathrm{~mm}$, the nozzle temperature $\mathrm{T}=210$, and the printing speed $\mathrm{F}=400 \mathrm{~mm} / \mathrm{min}$. The blood vessel stents has structural integrity and could meet the mechanical properties of the scaffold, as shown in Fig. 11.

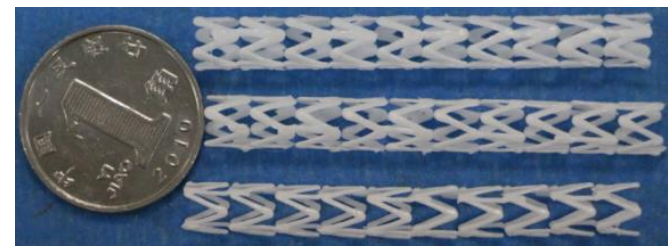

Fig. 11. 3D printed blood vessel stents.

\subsubsection{Experiment}

Through the transmission system and radiography, the bio degradable 3D printed vascular stent can be accurately implanted into the stenosis of pig vascular lesions and the stent implantation is successfully completed. From the perspective of B-ultrasound, the flow of stent and the blood flow were obviously improved, indicating that the stent was used to support the stenotic part of the stent. After 2 and 4 weeks of the stent implanting, the arterial color images were observed again, as shown in Fig. 12, in which the transverse coordinates expressed the time (s) and the longitudinal coordinates expressed the flow velocity (cm/s). Fig. 12 (a) is the photo after 2 weeks of stent implantation, color Doppler ultrasound showed stent flow was smooth. Fig. 12(b) shows that after 4 weeks of stenting, color Doppler ultrasound shows that the stent blood vessels are still open, but the blood flow signal is weaker than before. This may be related to the stent vascular stenosis after the stent is completely endothelialized. The specimens were taken out one month, two months and three months after the stent implantation. The femoral artery in the stent implantation did not have any restenosis or intimal hyperplasia, and the stent still had the function of supporting blood vessels.

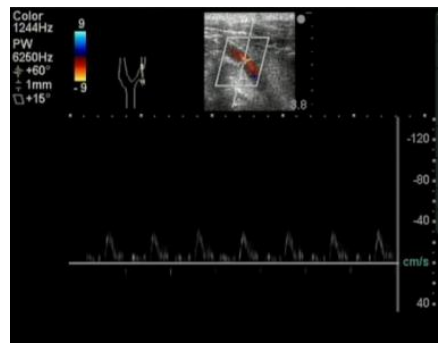

(a)

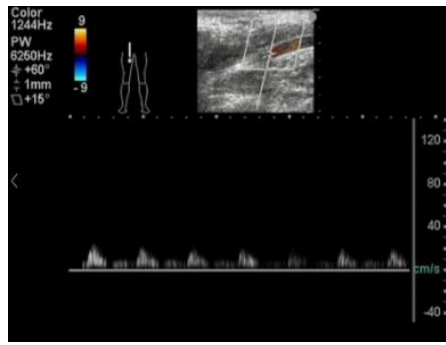

(b)

Fig. 12. Arterial color images after 2 and 4 weeks after stent implantation.

\section{Conclusion}

In this paper, in view of the problems in the design and manufacturing of biodegradable vascular scaffolds, a three-dimensional printing and molding equipment with independent intellectual property of PLA stent was developed. The three-dimensional model of the stent was designed and the mechanical properties were analyzed by the finite element method. The effects of the parameters of the nozzle geometry, the nozzle temperature and the printing speed on the surface morphology and mechanical properties of the stent were studied. The experimental results show that the printed blood vessel has good biocompatibility and biodegradability. It has important theoretical and experimental significance for the clinical transformation of biodegradable stent. 


\section{Acknowledgment}

The animal experiment in this paper was supported by Professor Zhou Min from Nanjing Gulou Hospital. This work was also supported by the Natural Science Foundation of Jiangsu Province, Natural Science Foundation of China (No. 51705259) Research funding of Nanjing Normal University (184080H202B135).

\section{References}

[1] Ontario, H. Q. (2010). Stenting for peripheral artery disease of the lower extremities: An evidence-based analysis. Ontario Health Technology Assessment, 10(18), 1-88.

[2] Ayodele, B. E. (2015). Modeling of FDM 3D printing for improved performance. Dissertations \& Theses-Gradworks, 15(2), 105-108.

[3] Liu, Q., Wang, Z., \& Lau, W. K. (2010). Methods and apparatus for fabricating porous 3D tubular scaffolds.

[4] Park, S. A., Lee, S. J., \& Lim, K. S. (2015). In vivo evaluation and characterization of a bio-absorbable drug-coated stent fabricated using a 3D-printing system. Materials Letters, 141, 355-358.

[5] Wang, W., Wang, L., Yang, D., et al. (2008). Design optimization of endovascular stent by finite element method. Journal of Biomedical Engineering, 25(2), 372.

[6] Ayodele, B. E. (2015). Modeling of FDM 3D printing for improved performance. Dissertations \& Theses-Gradworks, 15(2), 105-108.

[7] Inkinen, S., Hakkarainen, M., Albertsson, A. C., et al. (2015). From lactic acid to poly(lactic acid) (PLA): Characterization and analysis of PLA and its precursors. Biomacromolecules, 12(3), 523-532.

[8] Eshghi, N., Hojjati, M. H., Imani, M., et al. Finite element analysis of mechanical behaviors of coronary stent. Procedia Engineering, 10(2), 3056-3061.

[9] Tsai, M. H., Ou, K. L., Huang, C. F., et al. (2008). Study on micro-injection molding of light guiding plate by numerical simulation. International Communications in Heat \& Mass Transfer, 35(9), 1097-1100.

[10] Pang, X., Zhuang, X., Tang, Z., et al. (2010). Polylactic acid (PLA): Research, development and industrialization. Biotechnology Journal, 15(11), 1125-1136.

[11] Inkinen, S., Hakkarainen, M., Albertsson, A. C., et al. (2011). From lactic acid to poly(lactic acid) (PLA): Characterization and analysis of PLA and its precursors. Biomacromolecules, 12(3), 523-532.

[12] Barragan, P., Rieu, R., Garitey, V., et al. (2000). Elastic recoil of coronary stents: A comparative analysis. Catheterization and Cardiovascular Interventions, 50(1), 112.

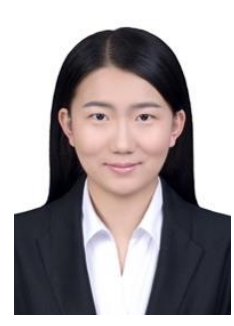

Zewei Xu received the B.S. degree from Nanjing Normal University, China, in 2016. She is currently pursuing the M.E. degree with Nanjing Normal University and the Jiangsu Key Laboratory of 3D printing equipment and manufacturing. Her research interests include 3D bio-printing for tissue engineering.

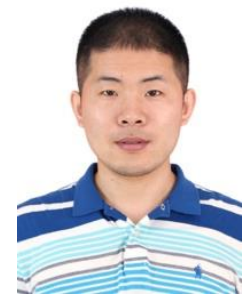

Zongan Li received the B.S. degree in mechanical engineering from the Nanjing University of Science and Technology, China, in 2009, and the Ph.D. degree in instrument science and technology from the Nanjing University of Science and Technology, China, in 2015. He was a post-doctoral researcher with Southeast University in 2016. He is a lecture in the Jiangsu Key Laboratory of 3D printing equipment and manufacturing of Nanjing Normal University since 2017. His current research interests include developing 3D model reconstruction 
software based on computed tomography images and 3-D bio-printing for tissue engine.

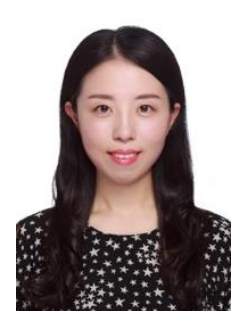

Liya Zhu received the B.S. degree from the Wuhan University of Technology, and the Ph.D. degree from the Nanjing University of Aeronautics and Astronautics, China. She is currently a lecturer with Nanjing Normal University and the Jiangsu Key Laboratory of 3D printing equipment and manufacturing. Her current research interests include 3-D bio-printing for tissue engineering.

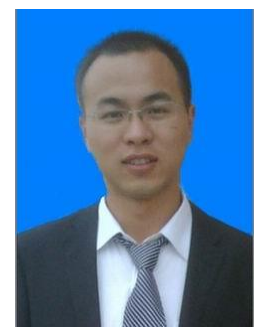

Jianping Shi received his B.S. degree in mechanical engineering in 2010 and received his M.S. degree in electronic engineering in 2013 from Nanjing Normal University, China. He received his Ph.D. degree in 2018 from Southeast University. He is now a lecturer at Nanjing Normal University and a researcher in the Jiangsu Key Laboratory for 3D printing equipment and manufacturing. His research interests include 3D printing methods and 3D bio-printing for tissue engineering.

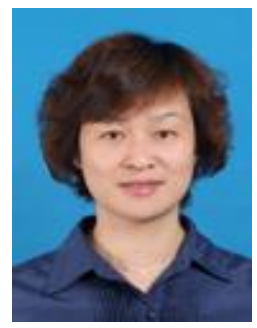

Chunmei Feng received her B.S. degree in Shandong University of Science and Technology in 1993 and received her M.S. degree in 2008 from Automation School of Nanjing University of Science and Technology, China. She is currently an associate professor at Nanjing Normal University and the Jiangsu Key Laboratory of 3D printing equipment and manufacturing. Her research interests include motor motion control.

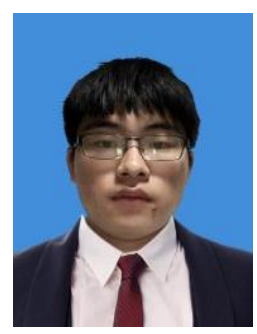

Zhe Yuan received his B.S. degree in electrical engineering and automation from JiangSu Normal University, JiangSu, China, in 2017. Currently, he is a master candidate in electrical engineering at Nanjing Normal University, JiangSu, China. 